\title{
Fabrication of polymer based integrated photonic devices by maskless lithography \\ M. Rahlves ${ }^{2}$ \\ ${ }^{a}$ Hannover Centre for Optical Technologies, Leibniz University Hannover, Nienburger Str. 17, D-30167 Hannover, Germany
}

\begin{abstract}
We present our recent results on the fabrication of photonic devices such as single-mode and few-mode waveguides, Ycouplers as well as integrated interferometric sensor devices. The devices were created by means of a fabrication method based on maskless lithography, which allows for fabricating embedded integrated polymer elements on a scale of several square centimeters with a resolution down to one micron. We demonstrate the versatility of our approach by presenting first results on photonic structures created by maskless lithography.
\end{abstract}

Keywords: Integrated photonics, Maskless lithography, Waveguides, Polymer photonics

\section{INTRODUCTION}

Integrated photonic devices have been used for a broad range of applications among which optical communication technology and optical sensing are the most prominent examples. Especially, the potential to fabricate these devices in a cost-effective manner enabled the market entry of low-cost sensing devices, for example, to trace biochemical analytes $[1,2]$. Lab-on-chip devices combining microfluidics and photonics are a promising alliance to determine analyte concentrations [2]. Low production cost may also pave the way to use these devices in medical applications which require low-cost disposables. The backbones of almost all photonic devices are light guiding structures such as optical waveguides. While the fabrication of optical waveguides was mainly done by semiconductor fabrication technologies in the past, the focus has recently been shifted to polymer based optics [3-9]. Polymers offer several advantages regarding fabrication cycles and, therefore, fabrication costs. In addition, there exist a vast range of technologies that generate microstructures in polymers to form waveguides and sensing elements depending on their specific field of operation and feature size. A process being capable of high-throughput rates based on reel-to-reel setups is hot embossing [3-5]. The process relies on a master stamp commonly made from silicon or metal which is transferred into a polymer substrate. Depending on the specific application of the device to be fabricated, all kinds of thermoplastics may be used as substrate materials. Common polymers used in hot embossing range from low priced polymethylmethacrylate (PMMA) to polyether ether ketone (PEEK) for high temperature applications. For waveguide fabrication, the embossing stamp and substrate material is usually heated above the glass transition temperature of the polymer and an embossing force is applied for a defined time. After cooling, the polymer substrate is released and contains grooves which are filled with a core material in a liquid monomer state. A rigid waveguide is formed by curing the monomer either thermally or by applying UV radiation [4,5]. Hot embossing is an adequate fabrication method for a high throughput rate but due to the required embossing stamp it is hardly adaptable to generate micro-geometries other than provided by the specific stamp. Therefore, printing of waveguides has drawn much attention during the last years. Both, flexographic and also inkjet printing, as one of the most versatile printing technology, were shown to be capable of creating optical waveguides with attenuations down to the few $\mathrm{dB} / \mathrm{cm}$ range [6,7]. If waveguides are to be operated in the single mode regime, twophoton-polymerization (2PP) also serves as a very adaptable method to generate optical elements and waveguides inside a liquid monomer [8]. However, 2PP is hardly mass fabrication compatible and is restricted to research-and-development applications. When using liquid monomers, another waveguide fabrication process was demonstrated where an ultraviolet laser beam is launched into the monomer and creates a polymerization seed point. Due to a self-focusing effect, the laser beam propagates linearly through the monomer and forms a rigid waveguide core. A rigid cladding can be formed by a subsequent flood exposure also utilizing ultraviolet light [9].

*maik.rahlves@hot.uni-hannover.de; phone+49 511 76217901; fax +49 511 76217909; www.hot.uni-hannover.de

Organic Photonic Materials and Devices XVIII, edited by Christopher E. Tabor, François Kajzar,

Toshikuni Kaino, Yasuhiro Koike, Proc. of SPIE Vol. 9745, 97450D - ( 2016 SPIE

CCC code: $0277-786 X / 16 / \$ 18 \cdot$ doi: $10.1117 / 12.2213211$

Proc. of SPIE Vol. 9745 97450D-1 


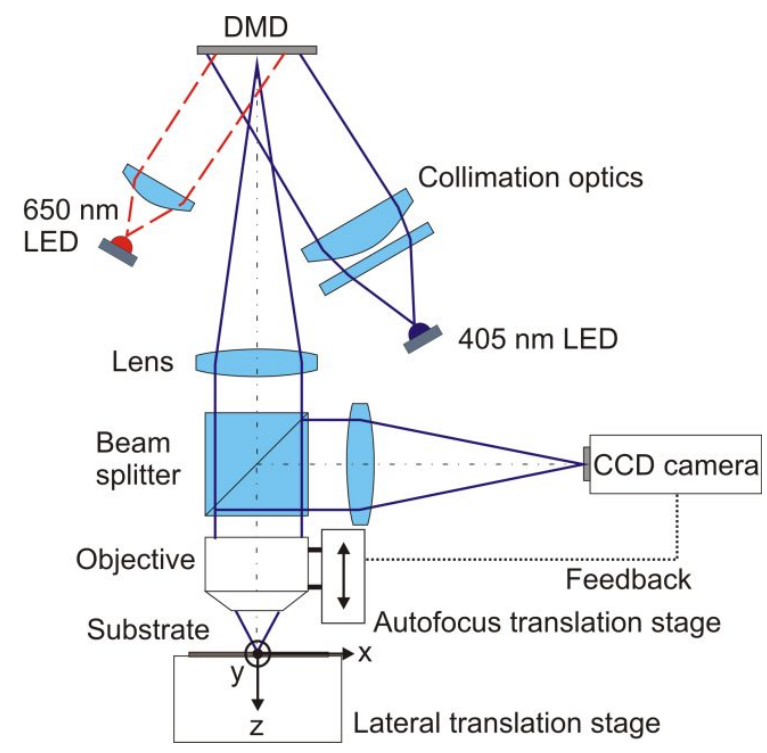

Figure 1. Sketch of the maskless lithographic setup utilized for waveguide fabrication.

To breach the gap between mass-fabrication compatible technologies and small-series research-and-development fabrication methods, we present our recent results on the fabrication of photonic devices such as single-mode and few mode waveguides, Y-couplers as well as integrated interferometric sensor devices by a simple maskless lithographic setup. To generate light guiding structures and sensor elements, we utilized a maskless lithography setup, which consists of a conventional microscope and a spatial light modulator (SLM). The SLM generates a pattern of the desired photonic structure, which is projected onto the substrate coated with photosensitive monomer. Using a light emitting diode with a center wavelength of $405 \mathrm{~nm}$, we are able to polymerize the photosensitive polymer in a desired pattern, which forms the cores of the waveguides. In future applications, we aim at the fabrication of small custom-made lab-on-chip devices for biomedical applications and chemical sensing.

\section{MASKLESS LITHOGRAPHIC SETUP}

To create structures in a liquid hybrid monomer to form optical elements, we utilized a self-developed maskless lithographic setup as shown in Fig. 1, similar to the one in [10]. The pattern of the microstructure or optical elements to be fabricated is generated by a digital-mirror-device (Texas Instruments DLP7000) used as SLM with a pixel pitch of $13.68 \mu \mathrm{m}$ and a total pixel number of $1024 \times 768$. For the lithographic process itself, we utilized a high power light emitting diode (LED) with a maximal output power of $0.9 \mathrm{~W}$ and a center wavelength of $405 \mathrm{~nm}$, which corresponds to the h-line of a mercury vapor lamp. To determine the optimal focal position, we included an additional LED which runs at a center wavelength of $650 \mathrm{~nm}$. Photoresists and even optically used monomers are commonly not sensitive in this wavelength region such that illuminating at $650 \mathrm{~nm}$ does not lead to a change of the photoresist or monomer. This enables one to view the substrate while finding the best focal position for lithography without altering the substrate or photoresist. Both LEDs are controlled via an Arduino microcontroller board. Since the pixel pitch of the digital-mirrordevice (DMD) is too large for the fabrication of single mode waveguides, we used a standard microscope setup (Carl Zeiss Microscopy) to de-magnify the DMD and to project its pattern onto the substrate. For our experiments, we used a microscope objective (Carl Zeiss EC Epiplan Apochromate) with a numerical aperture (NA) of 0.3 and a magnification of ten. In addition, the setup contains an infinity corrected tube lens provided by the same manufacturer. To determine the best focal position, we captured images of the substrate by a CCD camera (pike F421, AVT). The optical path of the imaging unit runs via a beam splitter and an additional tube lens as shown in Fig. 1. The best focal position was found automatically by moving the microscope objective in steps of $1 \mu \mathrm{m}$ in an interval of $500 \mu \mathrm{m}$ by using a piezoelectrically driven linear stage (SLC-1780-S, Smaract, Germany). By applying a variance based algorithm, we determined the microscope objective position which yielded the sharpest image and adjusted its position accordingly. The microscope field of view of $1.9 \mathrm{~mm} \times 1.1 \mathrm{~mm}$ is limited by the microscope setup and also restricts the maximum area which can be

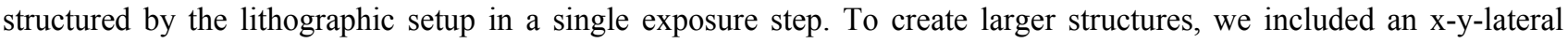
linear translation stage (two SLC-1780-S with orthogonally orientated axes, Smaract). This enables us to perform multiple exposures at different substrate areas sequentially which yields a maximal area used for the lithographic process of $5 \times 5 \mathrm{~cm}^{2}$. 
(1) Lithography

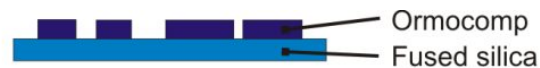

(2) Thermal bonding

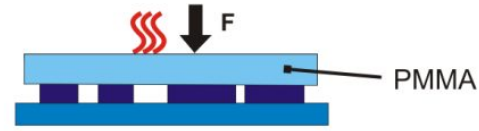

(3) Lift-off

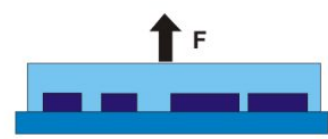

(4) Casting

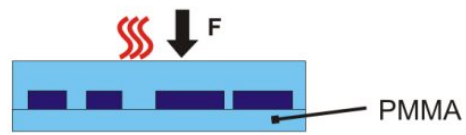

(1) Lithography

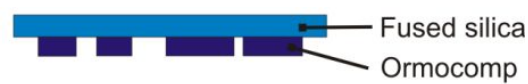

(2) Hot embossing
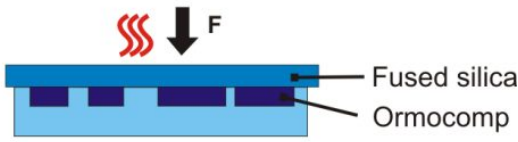

(3) Doctor blading

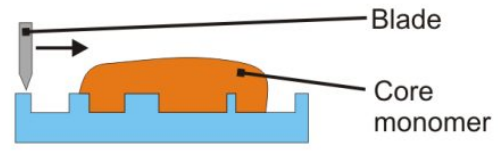

(4) Casting

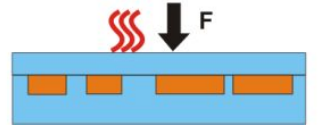

Figure 2. Process chains for fabrication of waveguides and optical structures: Direct structuring of monomers (left) and hot embossing based process (right).

\section{FABRICATION PROCESS}

The fabrication of waveguides and various integrated optical elements is based on an initial lithographic process step to form the optical structures. In a first step, a thin layer of Ormocomp (Microresist Technology, Germany) hybrid polymer was spin coated onto a microscope cover slide (Carl Zeiss) at a rotational speed of $4000 \mathrm{rpm}$. To achieve a coating thickness in the few micron range, $5 \mathrm{ml}$ Ormocomp was diluted using $5 \mathrm{ml}$ as well as $10 \mathrm{ml}$ of Ormothin thinner (Microresist Technology, Germany) to reduce its viscosity. After spin coating, we applied a prebake at $80^{\circ} \mathrm{C}$ for $2 \mathrm{~min}$ on a hot plate to vaporize the remaining thinner. For the lithographic setup, the cover slide was placed onto the lateral translation stage of the lithographic setup with the Ormocomp layer facing the microscope setup. The best focal position was found by switching on the red LED followed by an autofocus run. The actual lithographic process was carried out by uploading a bitmap image of the optical structure to be fabricated onto the DMD device and starting the exposure for 180 seconds. After exposure, we applied a second bake process step to the cover slide at $150^{\circ} \mathrm{C}$ for $10 \mathrm{~min}$. The cover slide was submerged into a developer bath (OrmoDev, Microresist Technology, Germany) for 1 min and rinsed with pure water subsequently.

To fabricate the final embedded waveguide structure, we pursued two different approaches. The first approach is based on a lift-off process where the cover slide including the optical structures is covered by a PMMA sheet (Evonik Industries) with thickness of $175 \mu \mathrm{m}$ as shown in Fig. 2 (left). Before carrying out the lift-off process step, the cover slide and the PMMA sheet are placed in a self-made hot embossing machine presented in [11] to thermally bond the Ormocomp structures to the PMMA sheet. The bonding process was carried out at a temperature of $150^{\circ} \mathrm{C}$ and a bonding force of $4 \mathrm{kN}$, which was kept for $5 \mathrm{~min}$. After cooling to room temperature, the PMMA sheet was separated from the cover slide such that the Ormocomp structures remained inside the PMMA layer to form the cores of the waveguide structures. To obtain embedded waveguides, an additional PMMA sheet with a thickness of $175 \mu \mathrm{m}$ was, again, thermally bonded onto the PMMA substrate layer utilizing the same process parameters as described previously.

The second approach for waveguide fabrication is based on a hot embossing process as shown in Fig. 2 (right) and similar to the one described in [4]. Here, the cover slide including the Ormocomp structures are only used as hot embossing stamp to transfer a negative copy of the structures obtained by the lithographic process into the PMMA sheet. To ensure that the Ormocomp structures remain on the cover slide during the entire process, a slightly lower embossing temperature of $130^{\circ} \mathrm{C}$ and force of $2 \mathrm{kN}$ is applied compared to the process described previously. After hot embossing, the substrate contains grooves which are filled with a liquid monomer (390119 UV Supraflex, Jänecke+Schneemann Druckfarben, Germany) to form the waveguide cores. To achieve an optically smooth waveguide surface and to avoid an 
unfavorable residual layer on top of the PMMA sheet, which would prevent or decrease waveguiding, we applied a manually performed doctor blading process.

To demonstrate the capability of the process, we fabricated arrays of linear optical waveguides in the few mode range. In addition, we created Y-splitters and Mach-Zehnder interferometers. The number of modes propagating inside the waveguides depends, in general, on the refractive indices of core and cladding and the waveguide geometry. PMMA with a refractive index of $n_{\text {clad }}=1.49$ in the visible range of the light spectrum servers as cladding material for the waveguides fabricated by both approaches. In case of the directly structured waveguides, Ormocore with a refractive index of $n_{\text {core }}=1.51$ forms the waveguide cores. Using the hot embossing process, the core material has a refractive index of $n_{\text {core }}=1.52$. To calculate the number of modes propagating inside a rectangular waveguide, we used the relation given in [12]:

$$
M=\pi \cdot\left(\frac{d}{\lambda}\right)^{2} \cdot N A^{2}
$$

where $M$ denotes the number of modes and $d$ the width and height of the waveguides assuming a rectangular crosssection. The vacuum wavelength is given by $\lambda$ and

$$
N A=\sqrt{n_{\text {core }}^{2}-n_{\text {clad }}^{2}} \text {. }
$$

Eqn. 2 yields an $N A=0.24$ and $N A=0.30$ for Ormocomp and Supraflex cores, respectively. To achieve single mode waveguides, $M$ needs to be in the interval $[1,2)$. Using Eqn. 1, we can estimate the core diameter $d$ to obtain singlemode waveguides assuming a wavelength of $633 \mathrm{~nm}$. This yields core dimensions in the interval $[1.46,2.06) \mu \mathrm{m}$ and $[1.19,1.68) \mu \mathrm{m}$ for both core materials, respectively. Hence, considering a feature size of $1.35 \mu \mathrm{m}$, which can be created by our setup, we are able to fabricate optical structures in the single mode regime. However, for preliminary experiments we restrict ourselves to few-mode waveguides with core diameters of $8.1 \mu \mathrm{m}$ which corresponds to a waveguide width of 6 DMD pixels.

\section{RESULTS}
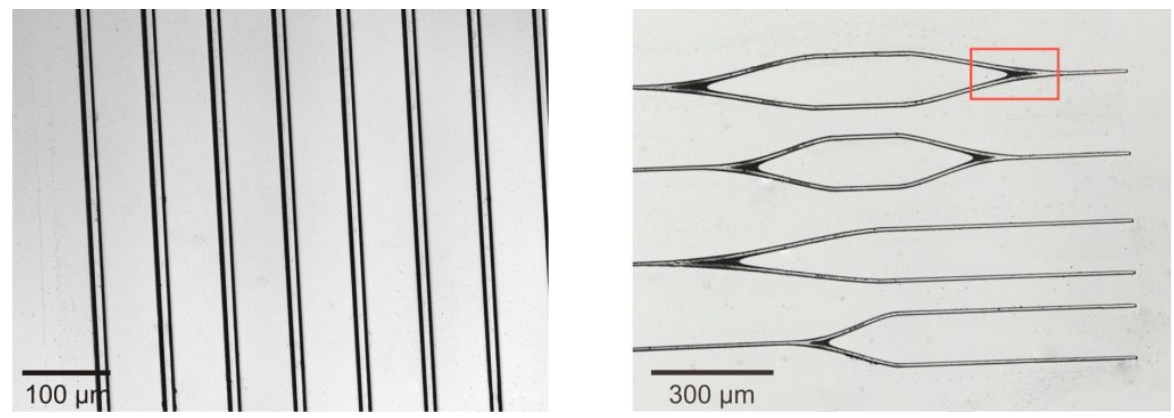

Figure 3. Microscope images of Ormocomp structures on fused silica cover slide fabricated by maskless lithography: linear waveguide array (left) and Y-splitter and Mach-Zehnder interferometers (right); the red box indicates the area shown in Fig. 4.

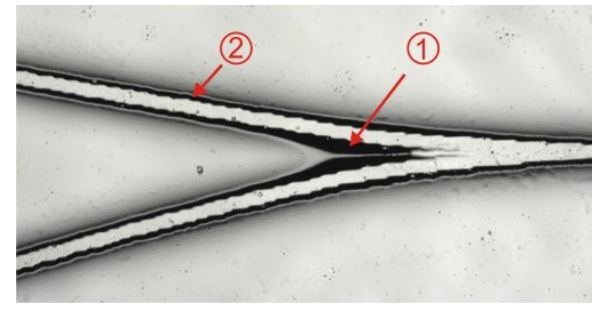

Figure 4. Enlargement of the image area indicated by the red box in Fig. 3 (right); side wall roughness of the waveguide due to resolved DMD pixel (1) and defect inside the splitter region due to the developer process (2). 


\subsection{Lithographic structuring}

Fig. 3 (left) shows a microscope image of a linear Ormocomp waveguide array and Fig. 3 (right) Y-splitters and MachZehnder interferometers fabricated on a fused silica cover slide. All structures presented in Fig. 3 were obtained using an Ormocomp-to-Ormothin ratio of 1:1. The enlargement depicted in Fig. 4 corresponds to the area indicated by the red box in Fig. 3 (right). The results displayed in Figs. 3 and 4 show that our process is capable of producing optical elements such as linear waveguides and more complex structures such as splitters and interferometers. However, Fig. 4 reveals that the splitter region exhibits periodic defects as indicated by (2) in Fig. 4. In addition, the junction region between the two waveguides shows defects due to the development process marked (1) in Fig. 4, which needs to be improved in future work.

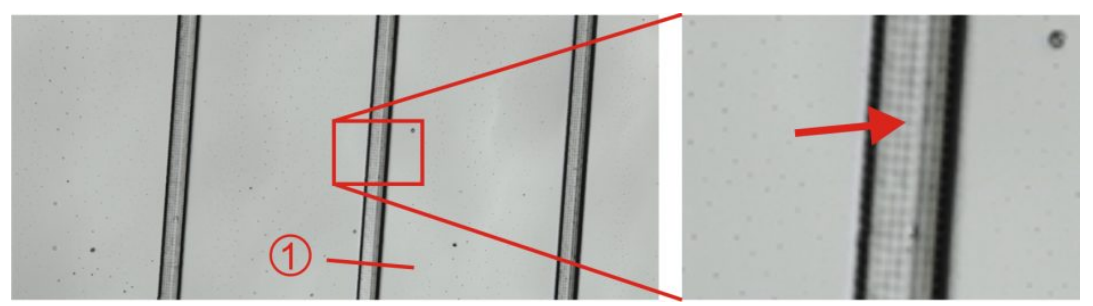

Figure 5. Microscope image of the fabricated Ormocomp waveguides on the microscope cover slide.

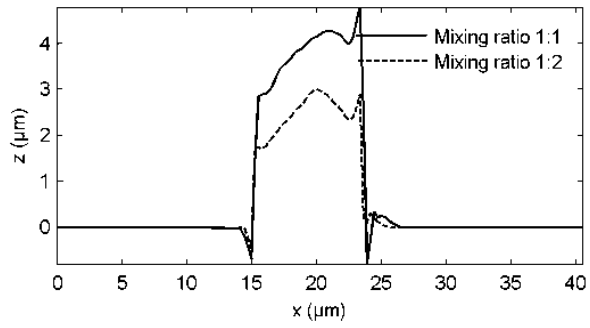

Figure 6. Confocal topography measurements along the red line indicated by (1) in Fig. 5 obtained on samples fabricated using an Ormocomp-to-Ormothin ratio of 1:1 and 1:2.

To investigate the structure height of the fabricated optical elements, we carried out confocal topography measurements (Keyence VK9600) in the measuring field displayed in Fig. 5 (right). The measurements were performed using samples which were fabricated with both Ormocer-to-Ormothin mixing ratios of 1:1 and 1:2. After development, we observed a remaining checkboard-like nanostructure, which is indicated by the red arrow in Fig. 5 (right). These periodic features are due to DMD pixels which are resolved by the microscope setup used or lithography and cause a waviness of a period of $1.35 \mu \mathrm{m}$. The confocal measurements yielded amplitudes of $20 \mathrm{~nm}$. To reduce this waviness, future work will include a reduction of the DMD pixel pitch as well as lithographic runs utilizing lenses with higher NA.

Cross-sections of the fabricated waveguides obtained by confocal microscopy are shown in Fig. 6. Note that the spikes at the edges of the waveguides are optical artifacts generated by the confocal microscope. In summary, the optical waveguides exhibit approximately rectangular cross-sections with a remaining curvature on top. We determined a maximum waveguide height of $2.9 \mu \mathrm{m}$ and $4.3 \mu \mathrm{m}$ for an Ormocomp-to-Ormothin mixing ratio of 1:2 and 1:1, respectively.

\subsection{Waveguide fabrication}

To create optical waveguides we carried out the experimental methods presented in Sec. 2. We found that the lift-off process does not yield satisfactory results using the current experimental parameters as described in Sec. 2. Under current conditions, only a fraction of the Ormocomp structures are transferred completely into the PMMA substrate leading to optical waveguides with intermittent discontinuities. Hence, these structures are, at the current stage, not suitable as optical waveguides. In future work, we will carry out an extensive parameter study regarding embossing and lift-off force as well as embossing and lift-off temperature to improve the process. 


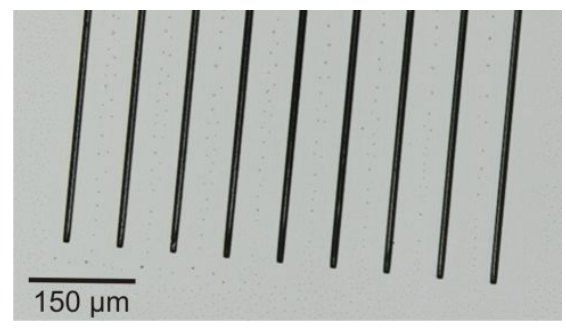

Figure 7. Microscope image of groove structures hot embossed into a PMMA substrate.

However, using the cover slide with Ormocomp structure as hot embossing stamp we achieved reasonably good results as shown in Fig. 7. Confocal measurement data of the grooves yielded a groove depth of $2.1 \mu \mathrm{m}$. The grooves were filled with Supraflex liquid monomer and cured by UV flood exposure. Systematic optimization of the process as well as optical attenuation measurements using the cut-back method will be carried out in future work.

\section{SUMMARY}

We presented a process chain for the fabrication of optical structures in polymers utilizing a versatile maskless lithographic process. In an initial process step, waveguides, Y-splitters and Mach-Zehnder interferometers were fabricated by projecting a bitmap image of the device to be fabricated onto a microscope cover slide coated with Ormocer hybrid polymer. After development of the Ormocer, waveguide structures were obtained with feature sizes in the few micron range. In a next step, the cover slide was used as hot embossing stamp to transfer the optical waveguides and sensing elements into a polymethylmethacrylate substrate, which was filled with a waveguide core material subsequently. In this work, we demonstrated the capability of the process to fabricate waveguides and sensing elements in the single mode regime considering a wavelength of $633 \mathrm{~nm}$. In the next steps, we will carry out optical measurements including waveguide attenuation, splitting ratio of Y-splitters as well as interferometric contrast of Mach-Zehnder interferometers. For future applications, our process enables a fast and versatile fabrication of waveguide based sensing elements in fields such as biomedical sensing. The high versatility of our approach allows a fast fabrication of proof-ofconcept sensor structures which is of high interest in both research and development.

\section{ACKNOWLEDGEMENTS}

This work was supported by the German Research Foundation in the framework of the special research center TRR/SFB 123 planar optronic systems (PlanOS).

\section{REFERENCES}

[1] Kelb, C., Rahlves, M., Reithmeier, E. and Roth, B.,"Realization and performance of an all-polymer optical planar deformation sensor," Sensors Journal, IEEE PP, 1-1 (2015)

[2] Kuswandi, B., Nuriman, Huskens, J. and Verboom, W., "Optical sensing systems for microfluidic devices: A review," Anal. Chim. Acta 601(2), 141-155 (2007)

[3] Worgull, M. [Hot Embossing - Theory and Technology of Microreplication], William Andrew, Oxford \& Burlington (2009)

[4] Rezem, M., Günther, A., Rahlves, M., Roth, B. and Reithmeier, E., "Hot Embossing of Polymer Optical Waveguides for Sensing Applications," Procedia Technology 15, 514-520 (2014)

[5] Choi, C.-G., "Fabrication of optical waveguides in thermosetting polymers using hot embossing," $J$. Micromech. Microeng. 14, 945-949 (2004)

[6] de Gans, B.-J., Duineveld, P.-C. and Schubert, U.-S., "Inkjet printing of polymers: State of the art and future developments," Advanced Materials 16, 203-213 (2004)

[7] Chappell, J., Hutt, D. and Conway, P., "Variation in the line stability of an inkjet printed optical waveguideapplicable material," in Electronics System-Integration Technology Conference, ESTC 2008. 2nd, 1267-1272 (2008) 
[8] Klein, S., Barsella, A., Leblond, H., Bulou, H., Fort, A., Andraud, C., Lemercier, G., Mulatier, J. C. and Dorkenoo, K., "One-step waveguide and optical circuit writing in photopolymerizable materials processed by two-photon absorption," Appl. Phys. Lett. 86, 211118 (2005)

[9] Günther, A., Petermann, A. B., Gleissner, U., Hanemann, T., Reithmeier, E., Rahlves, M., MeinhardtWollweber, M., Morgner, U. and Roth, B., "Cladded self-written multimode step-index waveguides using a one-polymer approach," Opt. Lett. 40, 1830-1833 (2015)

[10] Rahlves, M., Rezem, M., Boroz, K., Schlangen, S., Reithmeier, E. and Roth B., "A flexible, fast, and low-cost production process for polymer based diffractive optics," Opt. Express 23(3), 3618-3622 (2015)

[11] Rahlves, M., Kelb, C., Rezem, M., Schlangen, S., Boroz, K., Gödeke, D., Ihme, M. and B. Roth, B., "Digital mirror devices and liquid crystal displays in maskless lithography for fabrication of polymer-based holographic structures," J. Micro. Nanolithogr. MEMS MOEMS 14(4), 041302-1-8 (2015)

[12] Saleh, B. E. A. and Teich, M. C. [Fundamentals of Photonics], $2^{\text {nd }}$ edition, WILEY, Hoboken (2007) 